PROCEEDINGS OF THE

AMERICAN MATHEMATICAL SOCIETY

Volume 137, Number 1, January 2009, Pages 107-113

S 0002-9939(08)09479-3

Article electronically published on August 14, 2008

\title{
APPROXIMATION OF REAL NUMBERS WITH RATIONAL NUMBER SEQUENCES
}

\author{
RISTO KORHONEN
}

(Communicated by Ken Ono)

\begin{abstract}
Let $\alpha \in \mathbb{R}$, and let $C>\max \{1, \alpha\}$. It is shown that if $\left\{p_{n} / q_{n}\right\}$ is a sequence formed out of all rational numbers $p / q$ such that

$$
\left|\alpha-\frac{p}{q}\right| \leq \frac{1}{C q^{2}}
$$

where $p \in \mathbb{Z}$ and $q \in \mathbb{N}$ are relatively prime numbers, then either $\left\{p_{n} / q_{n}\right\}$ has finitely many elements or

$$
\limsup _{n \rightarrow \infty} \frac{\log \log q_{n}}{\log n} \geq 1
$$

where the points $\left\{q_{n}\right\}_{n \in \mathbb{N}}$ are ordered by increasing modulus. This implies that the sequence of denominators $\left\{q_{n}\right\}_{n \in \mathbb{N}}$ grows exponentially as a function of $n$, and so the density of rational numbers which approximate $\alpha$ well in the above sense is relatively low.
\end{abstract}

It has been suggested that one could obtain strong results in number theory by translating the proofs from Nevanlinna theory to number theory using, for instance, Vojta's dictionary [16]. Applying this method to Nevanlinna's proof of the second main theorem is said to yield strong results in number theory, including the $a b c$ conjecture formulated by Masser and Oesterlé. Difficulties arise, however, while trying to find a number-theoretic analogue for the ramification term in the second main theorem, since so far Vojta's dictionary does not have a translation for the derivative of a meromorphic function; see, e.g., 1, 7, 12.

There is a vast literature on the rational approximations of irrational numbers dating all the way back to Liouville [9], Thue [15], Siegel [13] and others (see [1] for further references). One of the fundamental results in the field is Roth's theorem, according to which for any algebraic irrational number $\alpha$ there are only finitely many solutions $p / q$ to

$$
\left|\alpha-\frac{p}{q}\right| \leq \frac{1}{q^{2+\varepsilon}}
$$

where $\varepsilon>0$, and $p$ and $q$ are relatively prime integers [11. Roth's theorem is sharp in the sense that the arbitrary $\varepsilon>0$ cannot be deleted in inequality (1). However, Lang [8] has proposed that if one would be able to follow the proof of the second main theorem with a good error term (see, e.g., [6]) to prove Roth's theorem, then

Received by the editors October 22, 2007, and, in revised form, January 9, 2008.

2000 Mathematics Subject Classification. Primary 11J68; Secondary 11J97.

The research reported in this paper was supported in part by the Academy of Finland grants \#118314 and \#210245.

(C)2008 American Mathematical Society 107 
one might be able to replace the quantity $1 / q^{2+\varepsilon}$ by $1 /\left(q^{2}(\log q)^{1+\varepsilon}\right)$. Even though this still remains as a conjecture, something can nevertheless be said. According to the Cugiani-Mahler theorem (see Bombieri and van der Poorten [2] for the latest version of the theorem), replacing the factor $q^{\varepsilon}$ in (11) with a certain sufficiently slowly decreasing function of $q$ yields a lower bound for the height growth of the solution sequence.

Osgood 10, and, independently, Steinmetz 14] generalized the weak second main theorem to moving target functions. Instead of making use of estimates on logarithmic derivatives, Steinmetz's method of proof is based on the second main theorem for holomorphic curves, which, according to Vojta's dictionary, corresponds to Schmidt's subspace theorem [16]. Using this fact, Vojta translated Steinmetz's proof to obtain a generalization of Roth's theorem where the target algebraic numbers $\alpha$ are allowed to vary slowly [17].

Halburd and the author have introduced a difference analogue of the second main theorem of Nevanlinna theory for meromorphic functions of finite order [4]. In this version of the theorem the counterpart of the ramification term depends on the exact difference operator $\Delta f=f(z+1)-f(z)$ for which there is a straightforward discrete analogue. The purpose of this paper is to use certain Nevanlinna theoretical ideas from [4, reinterpreted for convergent number sequences, in order to approximate arbitrary real numbers with sequences of rational numbers. Our findings are summarized in Theorem 11 below. The results obtained are disjoint from the Cugiani-Mahler theorem in the sense that both the assumption (2) and the assertion (3) of Theorem 11 are weaker than the corresponding statements in Cugiani-Mahler [2]. On the other hand, our method of proof enables us to include transcendental numbers.

Theorem 1. Let $\alpha \in \mathbb{R}$ and let $C>\max \{1, \alpha\}$. If $\left\{p_{n} / q_{n}\right\}$ is the sequence of all rational numbers $p / q$ such that

$$
\left|\alpha-\frac{p}{q}\right| \leq \frac{1}{C q^{2}}
$$

where $p \in \mathbb{Z}$ and $q \in \mathbb{N}$ are relatively prime numbers, then either the sequence $\left\{p_{n} / q_{n}\right\}_{n \in \mathbb{N}}$ contains finitely many elements or

$$
\limsup _{n \rightarrow \infty} \frac{\log \log q_{n}}{\log n} \geq 1
$$

where the sequence $\left\{q_{n}\right\}_{n \in \mathbb{N}}$ is ordered according to the increasing modulus of its elements.

Theorem 1 implies that if (2) has infinitely many solutions, then the denominators $q_{n}$ in the solution sequence of (2) grow faster than any polynomial of $n$, and so the density of rational numbers which approximate $\alpha$ well in the sense of (2) is relatively low. The fact that Theorem 1 does not rule out the possible existence of infinitely many solutions of (2) with exponentially fast growing denominators appears to correspond to the fact that the difference analogue of the second main theorem [4] (see [3] for an improved error term) has a larger error term than the original second main theorem if the order of the meromorphic function is at least one. For meromorphic functions of order strictly less than one, the error term in the difference analogue of the second main theorem is, however, smaller than in 
Nevanlinna's second main theorem. Therefore, since the second main theorem corresponds to Roth's theorem in Vojta's dictionary, and since the proof of Theorem 1 is based on estimating differences of number sequences, the fact that Theorem 1 gives sharper results than Roth's theorem in the special case where the sequence of rational numbers $\left\{p_{n} / q_{n}\right\}_{n \in \mathbb{N}}$ satisfies

$$
\limsup _{n \rightarrow \infty} \frac{\log \log q_{n}}{\log n}<1,
$$

that is, when the sequence $\left\{q_{n}\right\}_{n \in \mathbb{N}}$ is of "order less than one", is consistent with Vojta's dictionary.

Concerning the sharpness of Theorem 1 we refer to [5, pp. 163-165], where it is shown that if $\left\{F_{n}\right\}_{n \in \mathbb{N}}$ is the Fibonacci sequence, then at least one out of every three consecutive numbers, say $\left\{F_{n_{k}-1} / F_{n_{k}}\right\}_{k \in \mathbb{N}}$, in the sequence $\left\{F_{n-1} / F_{n}\right\}_{n \in \mathbb{N}}$ satisfies

$$
\left|\frac{1}{2}(\sqrt{5}-1)-\frac{F_{n_{k}-1}}{F_{n_{k}}}\right|<\frac{1}{\sqrt{5} F_{n_{k}}^{2}} .
$$

Therefore, in the notation of Theorem 1, $\alpha=(\sqrt{5}-1) / 2$ and $C=\sqrt{5}$, and so the assumption $C>\max \{1, \alpha\}$ is satisfied. Moreover, since

$$
F_{n}=\frac{(1+\sqrt{5})^{n}-(1-\sqrt{5})^{n}}{2^{n} \sqrt{5}}
$$

for all $n \in \mathbb{N}$, it immediately follows that

$$
\limsup _{k \rightarrow \infty} \frac{\log \log F_{n_{k}}}{\log n_{k}}=1,
$$

and so the lower bound (3) is attained for the sequence $\left\{F_{n_{k}-1} / F_{n_{k}}\right\}_{k \in \mathbb{N}}$.

Proof of Theorem 1. Suppose that the sequence $\left\{x_{n}\right\}_{n \in \mathbb{N}}$ of all distinct rational numbers $x_{n} \neq \alpha$ satisfying (2) contains infinitely many elements, since otherwise there is nothing to prove. Then, by defining $\log ^{+} x=\max \{0, \log x\}$ for all $x \geq 0$, it follows that

$$
\begin{aligned}
\log ^{+}\left|\frac{1}{\alpha-x_{n}}\right| & \leq \log ^{+}\left|\frac{x_{n+1}-x_{n}}{\alpha-x_{n}}\right|+\log ^{+}\left|\frac{1}{x_{n+1}-x_{n}}\right| \\
& =\log ^{+}\left|1-\frac{x_{n+1}-\alpha}{x_{n}-\alpha}\right|+\log ^{+}\left|\frac{1}{x_{n+1}-x_{n}}\right|
\end{aligned}
$$

for all $n \in \mathbb{N}$. We may write $\left\{x_{n}\right\}_{n \in \mathbb{N}}=\left\{y_{n}\right\}_{n \in \mathbb{N}} \cup\left\{z_{n}\right\}_{n \in \mathbb{N}}$, where $y_{n}>\alpha$ and $z_{n}<\alpha$ for all $n \in \mathbb{N}$, and we have rearranged the terms in these sequences such that $\alpha<y_{n+1}<y_{n}$ and $\alpha>z_{n+1}>z_{n}$ for all $n \in \mathbb{N}$. Therefore,

$$
0<\frac{y_{n+1}-\alpha}{y_{n}-\alpha}<1 \text { and } 0<\frac{\alpha-z_{n+1}}{\alpha-z_{n}}<1
$$

and so

$$
\log ^{+}\left|1-\frac{y_{n+1}-\alpha}{y_{n}-\alpha}\right|=0 \quad \text { and } \quad \log ^{+}\left|1-\frac{z_{n+1}-\alpha}{z_{n}-\alpha}\right|=0
$$

for all $n \in \mathbb{N}$. By combining (44) and (5), it follows that

$$
\log ^{+}\left|\frac{1}{\alpha-y_{n}}\right| \leq \log ^{+}\left|\frac{1}{y_{n+1}-y_{n}}\right| \quad \text { and } \quad \log ^{+}\left|\frac{1}{\alpha-z_{n}}\right| \leq \log ^{+}\left|\frac{1}{z_{n+1}-z_{n}}\right|
$$


for all $n \in \mathbb{N}$. By assumption at least one of the sequences $\left\{y_{n}\right\}_{n \in \mathbb{N}}$ and $\left\{z_{n}\right\}_{n \in \mathbb{N}}$ contains infinitely many elements. If $\#\left\{y_{n}\right\}_{n \in \mathbb{N}}=\infty$, then, by denoting $y_{n}=$ $a_{n} / b_{n}$, it follows that

$$
\begin{aligned}
\log ^{+}\left|\frac{1}{\alpha-y_{n}}\right| & \leq \log ^{+}\left|\frac{b_{n} b_{n+1}}{a_{n+1} b_{n}-a_{n} b_{n+1}}\right| \\
& =\log \max \left\{\left|b_{n} b_{n+1}\right|,\left|a_{n+1} b_{n}-a_{n} b_{n+1}\right|\right\}-\log \left|a_{n+1} b_{n}-a_{n} b_{n+1}\right| \\
& =\log ^{+}\left|\frac{a_{n+1} b_{n}-a_{n} b_{n+1}}{b_{n} b_{n+1}}\right|+\log \left|b_{n} b_{n+1}\right|-\log \left|a_{n+1} b_{n}-a_{n} b_{n+1}\right| \\
& =\log ^{+}\left|y_{n+1}-y_{n}\right|+\log \left|b_{n} b_{n+1}\right|-\log \left|a_{n+1} b_{n}-a_{n} b_{n+1}\right|
\end{aligned}
$$

for all $n \in \mathbb{N}$. Since $\left\{y_{n}\right\}_{n \in \mathbb{N}}$ is a decreasing sequence of $n$, it follows that

$$
\log ^{+}\left|y_{n+1}-y_{n}\right| \leq \log ^{+}\left|\frac{y_{n+1}}{y_{n}}-1\right|+\log ^{+}\left|y_{n}\right|=\log ^{+}\left|y_{n}\right|
$$

for all $n \in \mathbb{N}$, and so (6) yields

$$
\log ^{+}\left|\frac{1}{\alpha-y_{n}}\right| \leq \log ^{+}\left|y_{n}\right|+\log \left|b_{n} b_{n+1}\right| .
$$

Since, by (2),

$$
\log ^{+}\left|\frac{1}{\alpha-y_{n}}\right| \geq 2 \log \left|b_{n}\right|+\log |C|
$$

for all $n$ sufficiently large, it follows by combining inequalities (77) and (8) that

$$
\log \left|b_{n}\right|+\log |C| \leq \log ^{+}\left|y_{n}\right|+\log \left|b_{n+1}\right| .
$$

Therefore, by the assumption $C>\max \{1, \alpha\}$, the sequence $\left\{b_{n}\right\}_{n \in \mathbb{N}}$ is increasing for all sufficiently large $n$. Now, since both $\left\{b_{n}\right\}_{n \in \mathbb{N}}$ and $\left\{q_{n}\right\}_{n \in \mathbb{N}}$ are non-decreasing sequences of $n$, and $\left\{b_{n}\right\}_{n \in \mathbb{N}} \subset\left\{q_{n}\right\}_{n \in \mathbb{N}}$, it follows that

$$
\limsup _{n \rightarrow \infty} \frac{\log \log q_{n}}{\log n} \geq \limsup _{n \rightarrow \infty} \frac{\log \log b_{n}}{\log n} .
$$

Moreover, if

$$
\limsup _{n \rightarrow \infty} \frac{\log \log b_{n}}{\log n}<1
$$

then Lemma 2 below implies that

$$
\log |C| \leq \log ^{+}\left|y_{n}\right|+o(1),
$$

where $n$ runs to infinity outside of an exceptional set $F$ satisfying $\sum_{F} n^{-1}<\infty$. This is in contradiction with the assumption $C>\max \{1, \alpha\}$, and so (9) yields

$$
\limsup _{n \rightarrow \infty} \frac{\log \log q_{n}}{\log n} \geq \limsup _{n \rightarrow \infty} \frac{\log \log b_{n}}{\log n} \geq 1 \text {. }
$$

If $\#\left\{z_{n}\right\}_{n \in \mathbb{N}}=\infty$, then by using the fact that $\left\{z_{n}\right\}_{n \in \mathbb{N}}$ is an increasing sequence, it follows similarly as above that

$$
\log ^{+}\left|z_{n+1}-z_{n}\right| \leq \log ^{+}\left|\frac{z_{n}}{z_{n+1}}-1\right|+\log ^{+}\left|z_{n+1}\right|=\log ^{+}\left|z_{n+1}\right|
$$


for all $n \in \mathbb{N}$. Hence, by denoting $z_{n}=c_{n} / d_{n}$ and using similar argumentation as above, it follows that

$$
\log \left|d_{n}\right|+\log |C| \leq \log ^{+}\left|z_{n+1}\right|+\log \left|d_{n+1}\right|,
$$

whenever $n$ is sufficiently large. Therefore also $\left\{d_{n}\right\}_{n \in \mathbb{N}}$ is increasing for all sufficiently large $n$, and so Lemma 2 yields (10) in the case

$$
\limsup _{n \rightarrow \infty} \frac{\log \log d_{n}}{\log n}<1
$$

This is again in contradiction with the assumption $C>\max \{1, \alpha\}$, and therefore

$$
\limsup _{n \rightarrow \infty} \frac{\log \log q_{n}}{\log n} \geq \limsup _{n \rightarrow \infty} \frac{\log \log d_{n}}{\log n} \geq 1 .
$$

This concludes the proof of Theorem 1

Lemma 2. If $Q: \mathbb{N} \rightarrow[0,+\infty)$ is a non-decreasing function such that

$$
\limsup _{n \rightarrow \infty} \frac{\log \log Q(n)}{\log n}=\sigma<\infty
$$

and $\kappa \in(0,1)$, then

$$
\log Q(n+1)=\log Q(n)+o\left(\frac{\log Q(n)}{n^{\kappa}}\right)
$$

as $n$ approaches infinity outside of a set $F \subset \mathbb{N}$ satisfying $\sum_{n \in F} n^{-1}<\infty$.

Proof. Let $\delta \in(\kappa, 1)$ and assume that the set $F \subset \mathbb{N}$ defined by

$$
F=\left\{n \in \mathbb{N}: \frac{\log Q(n+1)-\log Q(n)}{\log Q(n)} \cdot n^{\delta} \geq 1\right\}
$$

satisfies $\sum_{n \in F} n^{-1}=\infty$. Denote $F=\bigcup_{k=0}^{\infty}\left\{n_{k}\right\}$, let $\varepsilon>0$, and suppose that there exists an $N \in \mathbb{N}$ such that $n_{k} \geq k^{1+\varepsilon}$ for all $k \geq N$. But then,

$$
\sum_{F} \frac{1}{n}=\sum_{k=1}^{\infty} \frac{1}{n_{k}} \leq \sum_{k=1}^{N} \frac{1}{n_{k}}+\sum_{k=N+1}^{\infty} \frac{1}{k^{1+\varepsilon}}<\infty,
$$

which contradicts the assumption $\sum_{F} n^{-1}=\infty$. Therefore the sequence $\left\{n_{k}\right\}_{k \in \mathbb{N}}$ has a subsequence $\left\{n_{k_{j}}\right\}_{j \in \mathbb{N}}$ such that $n_{k_{j}} \leq k_{j}^{1+\varepsilon}$ for all $j \in \mathbb{N}$. Since, by (13),

$$
\left(1+\frac{1}{n_{k}^{\delta}}\right) \log Q\left(n_{k}\right) \leq \log Q\left(n_{k+1}\right)
$$

for all $k \in \mathbb{N}$, it follows by iterating along the sequence $\left\{n_{k_{j}}\right\}_{j \in \mathbb{N}}$ that

$$
\log Q\left(n_{k_{j}}\right) \geq \prod_{\nu=0}^{k_{j}-1}\left(1+\frac{1}{n_{\nu}^{\delta}}\right) \log Q\left(n_{0}\right)
$$


for all $j \in \mathbb{N}$, and so, by choosing $\varepsilon$ such that $(1+\varepsilon) \delta<1$,

$$
\begin{aligned}
\limsup _{n \rightarrow \infty} \frac{\log \log Q(n)}{\log n} & \geq \limsup _{j \rightarrow \infty} \frac{\log \log Q\left(n_{k_{j}}\right)}{\log n_{k_{j}}} \\
& \geq \limsup _{j \rightarrow \infty} \frac{\log \log Q\left(n_{0}\right)+\sum_{\nu=0}^{k_{j}-1} \log \left(1+1 / n_{\nu}^{\delta}\right)}{\log n_{k_{j}}} \\
& \geq \limsup _{j \rightarrow \infty} \frac{\log \log Q\left(n_{0}\right)+k_{j} \log \left(1+1 / n_{k_{j}}^{\delta}\right)}{(1+\varepsilon) \log k_{j}}=\infty,
\end{aligned}
$$

which contradicts (11). Hence $\sum_{n \in F} n^{-1}$ must be finite; in other words,

$$
\log Q(n+1)=\log Q(n)+O\left(\frac{\log Q(n)}{n^{\delta}}\right)
$$

where $n$ runs to infinity outside of an exceptional set $F$ satisfying $\sum_{n \in F} n^{-1}<\infty$. Since $\delta>\kappa$, the assertion (12) follows.

\section{REFERENCES}

[1] E. Bombieri and W. Gubler, Heights in Diophantine geometry, New Mathematical Monographs, vol. 4, Cambridge University Press, Cambridge, 2006. MR.2216774 (2007a:11092)

[2] E. Bombieri and A. J. van der Poorten, Some quantitative results related to Roth's theorem, J. Austral. Math. Soc. Ser. A 45 (1988), no. 2, 233-248; Corrigendum: J. Austral. Math. Soc. Ser. A 48 (1990), no. 1, 154-155. MR0951583 (89i:11075) MR 1026847 (90m:11103)

[3] R. G. Halburd, R. Korhonen, and K. Tohge, Cartan's value distribution theory for Casorati determinants, preprint.

[4] R. G. Halburd and R. J. Korhonen, Nevanlinna theory for the difference operator, Ann. Acad. Sci. Fenn. Math. 31 (2006), 463-478. MR.2248826 (2007e:30038)

[5] G. H. Hardy and E. M. Wright, An introduction to the theory of numbers, The Clarendon Press, Oxford University Press, New York, 1979. MR568909 (81i:10002)

[6] A. Hinkkanen, A sharp form of Nevanlinna's second fundamental theorem, Invent. Math. 108 (1992), 549-574. MR.1163238 (93c:30045)

[7] P.-C. Hu and C.-C. Yang, Value distribution theory related to number theory, Birkhäuser Verlag, Basel, 2006. MR2245631

[8] S. Lang, The error term in Nevanlinna theory, Duke Math. J. 56 (1988), no. 1, 193-218. MR.932862 (89g:32037)

[9] J. Liouville, Sur des classes très-étendues de quantités dont la valeur n'est ni algébrique, ni même réductible à des irrationelles algébriques, Journal de Math. Pures et Appl. 16 (1851), 133-142.

[10] C. F. Osgood, Sometimes effective Thue-Siegel-Roth-Schmidt-Nevanlinna bounds, or better, J. Number Theory 21 (1985), 347-389. MR814011 (87f:11046)

[11] K. F. Roth, Rational approximations to algebraic numbers, Mathematika 2 (1955), 1-20. MR0072182(17:242d)

[12] M. Ru, Nevanlinna theory and its relation to Diophantine approximation, vol. 1052, World Scientific Publishing Co., Inc., River Edge, NJ, and Singapore, 2001. MR.1850002 (2002g:11106)

[13] C. L. Siegel, Approximation algebraischer Zahlen, Math. Z. 10 (1921), 173-213. MR.1544471

[14] N. Steinmetz, Eine Verallgemeinerung des zweiten Nevanlinnaschen Hauptsatzes, J. Reine Angew. Math. 368 (1986), 134-141. MR850619(87i:30056)

[15] A. Thue, Über Annäherungswerte algebraischer Zahlen, J. Reine Angew. Math. 135 (1909), 284-305. 
[16] P. Vojta, Diophantine approximations and value distribution theory, Lecture Notes in Math., vol. 1239, Springer-Verlag, Berlin, 1987. MR883451 (91k:11049)

[17] _ Roth's theorem with moving targets, Internat. Math. Res. Notices 1996 (1996), 109114. MR:1383752 (96k:11087)

Department of Physics and Mathematics, University of Joensud, P.O. Box 111, FI-80101 Joensuu, Finland

E-mail address: risto.korhonen@joensuu.fi

Current address: Department of Mathematics and Statistics, P.O. Box 68, FI-00014, University of Helsinki, Finland

E-mail address: risto.korhonen@helsinki.fi 